Document downloaded from:

http://hdl.handle.net/10251/102298

This paper must be cited as:

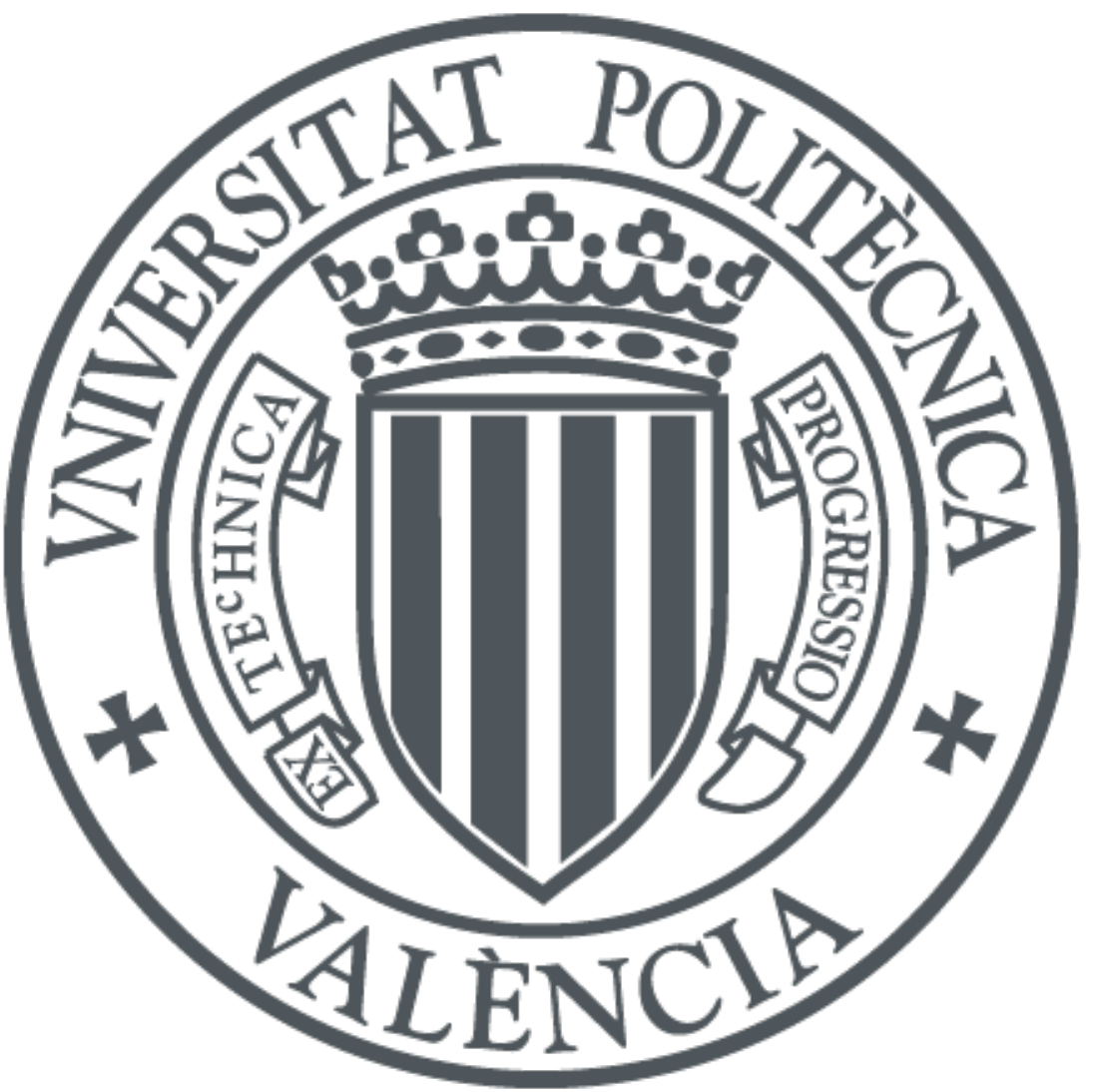

The final publication is available at

http://doi.org/10.1109/TAP.2017.2670368

Copyright Institute of Electrical and Electronics Engineers

Additional Information 


\section{A Comparison between Natural Resonances and Characteristic Mode Resonances of an infinite circular cylinder}

\author{
Tomás Bernabeu-Jiménez, Member, IEEE, \\ Alejandro Valero-Nogueira, Senior Member, IEEE, \\ Felipe Vico, Member, IEEE, \\ and Ahmed A. Kishk, Fellow, IEEE
}

\begin{abstract}
Here, some aspects in the interpretation of the solutions of a PEC infinite circular cylinder with the Theory of Characteristic Modes are presented. Firstly, natural resonances and characteristic mode resonances are introduced and compared. Secondly, characteristic eigenvalues are used to find those natural resonances considering complex $k a$ values. Furthermore, by linking the standard and the generalized eigenvalue problems a relation between natural resonances and characteristic mode eigenvalues is shown. Finally, the thesis stating that external characteristic mode resonance does not imply maximum field scattering, is also demonstrated.
\end{abstract}

Keywords-PEC cylinder, EFIE, natural resonances, eigenvalues and eigenfunctions, characteristic modes.

\section{INTRODUCTION}

Natural resonances are well known in the field of target identification. They are aspect independent, depending only on the intrinsic properties of the target and the surrounding media. This makes them a good tool for detection and discrimination in radar applications. In practice, natural resonances are extracted from the late time responses of impinging electromagnetic waves. This can be done using some well known theoretical procedures to extract the poles related with the target. Some of the main techniques for pole extraction are the Singularity Expansion Method (SEM) [1], the Prony's method [2], the Matrix Pencil (MP) method [3], and the Cauchy's method [4]. These methods are also used to extract poles from the radiated fields. An alternative approach, similar to SEM, would be to determine the natural resonances by searching for the zeros of the determinant of the Method of Moments (MoM) matrix in the complex frequency plane, [5], [6]. Or, equivalently, to find these zeros using a standard eigenvalue problem (SEP), where natural eigenmodes and natural eigenfrequencies are computed. This method is called eigenmode expansion method (EEM), and it was introduced by Baum in [7]. All methods mentioned above provide the same set of resonances. These resonances are in general complex numbers and are also known as complex natural resonances (CNRs). CNRs resonances can be classified into internal or external to the body under consideration. Internal resonances are merely cavity resonances caused by the internal waves experiencing multiple internal reflections. External resonances, however, are caused by creeping waves propagating along the body surface with attenuation due to the continuous radiation in the tangent direction. It is worth recalling that, unlike external, internal resonances are pure real numbers because they are undamped, representing those solutions that can not radiate outside the PEC cavity.

Along with these resonances, a different approach is being widely used lately to find other type of modes and resonances. This method is called Characteristic Mode Analysis (CMA). CMA was first proposed by Garbacz [8], and reformulated by Harrington, Mautz and Chang, establishing a more direct procedure to obtain Garbacz's modal expansion [9]-[11]. They considered integro-differential formulations and the associated impedance matrix of the Method of Moments (MoM) to compute the characteristic modes and their corresponding eigenvalues. CMA was initially proposed as an antenna synthesis

Manuscript received September 20, 2016 and optimization tool for antennas and scatterers and after [12], it gradually attracted more interest in the electromagnetic community as a help for resonant-size antenna design, given the physical insight it provides on the radiation mechanisms taking place in the antenna. Indeed, CMA eigensolutions possess interesting mathematical properties. Characteristic currents are orthogonal with respect to the real part of the impedance matrix operator. Therefore it is readily seen that corresponding characteristic fields constitute an orthonormal set. In addition, characteristic currents and their corresponding eigenvalues are real quantities, which facilitates their manipulation and interpretation. But, more interestingly, it is commonly accepted that CMA provides a physical insight through the eigenvalues and their corresponding characteristic currents. Characteristic eigenvalues are linked to the amount of energy stored by their corresponding modes. In this regard, CMA states that a given characteristic mode resonates at a frequency where the eigenvalue becomes zero.

However, there are still unknown aspects in the interpretation of the resulting characteristic mode resonances (CMRs). Interestingly, as for natural resonances, CMRs can also be classified into internal or external. As explained in [9], when a eigenvalue is zero or infinite, the corresponding characteristic mode is resonating externally or internally to the object, respectively. Therefore, in order to interpret these solutions, it is interesting to look at the natural resonances since they are unique, regardless of the technique considered to compute them or the formulation employed. [13].

Concerning PEC cavities, some efforts have been done in the past to stablish the relation between CNRs and CMRs. In [14] and [15] it was shown that internal cavity natural resonances coincide with internal CMRs. However, although external CMRs were also mentioned in [14], no discussion was provided on the relation between them and external CNRs. As for [15], wrong conlusions were drawn. Moreover, in [16] it was wrongly stated that CNRs are the zeros of the eigenvalues of the governing equation in CMA. Recently, Sarkar et al. published some insightful explanations about characteristic modes and its resonances [17]. Building on that analysis, we present here some additional statements supporting the ideas from Sarkar and coauthors.

For that purpose, in this work, some aspects in the interpretation of the Characteristic Mode solutions for a PEC infinite circular cylinder are presented. Firstly, natural resonances and characteristic resonances are compared. Secondly, it is observed that natural resonances can be obtained form characteristic eigenvalues when complex $k a$ plane is considered. Furthermore, by linking standard and generalized eigenvalue problems, a mathematical relation between natural resonances and characteristic eigenvalues is found. Finally, the thesis stating that external characteristic mode resonances does not imply maximum field scattering [17], is also demonstrated.

\section{Natural Mode vs Characteristic Mode Definition}

Consider a PEC scatterer illuminated by an incident electric field, $\mathbf{E}^{i}$. This incident field induces an electric current distribution $\mathbf{J}$ flowing on the PEC surface that radiates a scattered field $\mathbf{E}^{s}$. After imposing the boundary conditions for the tangential field on the PEC surface, and considering that the scattered field can be expressed as $\mathbf{E}^{s}=-Z(\mathbf{J})$ the problem to be solved can be written from the functional defined by the electric field integral equation (EFIE).

$$
Z(\mathbf{J}(\mathbf{r}, \omega))=\mathbf{E}^{i}(\mathbf{r}, \omega)
$$

Where the functional relation $Z$ is 


$$
\begin{aligned}
Z(\mathbf{J}(\mathbf{r}, \omega))=-\frac{j \omega \mu}{4 \pi} & \iint_{S} \mathbf{J}\left(\mathbf{r}^{\prime}\right) \frac{\mathrm{e}^{j k\left|\mathbf{r}-\mathbf{r}^{\prime}\right|}}{\left|\mathbf{r}-\mathbf{r}^{\prime}\right|} d S^{\prime} \\
& +\frac{1}{4 \pi j \omega \epsilon} \nabla \iint_{S} \nabla^{\prime} \mathbf{J}\left(\mathbf{r}^{\prime}\right) \frac{\mathrm{e}^{j k\left|\mathbf{r}-\mathbf{r}^{\prime}\right|}}{\left|\mathbf{r}-\mathbf{r}^{\prime}\right|} d S^{\prime}
\end{aligned}
$$

In (2) $\mathbf{r}$ denotes the observation point, $\mathbf{r}$ ' the source point, $S$ the scattering surface, $\omega$ the angular frequency, and $\mu, \epsilon$ and $k$ the permeability, permittivity, and the wavenumber, respectively. Equation (1) is solved for the current $J$ through the inverse of the impedance operator, $\mathbf{J}(\mathbf{r}, \omega)=Z^{-1} \mathbf{E}^{i}(\mathbf{r}, \omega)$.

The resonance problem considered in this work is formulated in (3), where no excitation is applied, i.e., $\mathbf{E}^{i}(\mathbf{r}, \omega)=0$. Equation (3) defines the natural frequencies and natural modes of the target involved in the scattering process,

$$
Z(\mathbf{J}(\mathbf{r}, \omega))=0
$$

meaning that one seeks for the nontrivial solutions of (3), which requires to find the solutions for which $Z$ is a singular operator. This generally occurs at an infinite number of discrete complex frequencies, known as natural frequencies, $\omega=\omega^{\prime}+j \omega^{\prime \prime}$. Where $\omega^{\prime}$ provides the natural resonant frequency of a given natural mode, and $\omega^{\prime \prime}$ its damping factor. The damping factor is linked to the radiating properties of a given mode.

To find the singularities of the $Z$ operator one can seek for the zeros of the eigenvalues of the standard eigenvalue problem, $\nu_{n}=0$. Or, equivalently, to solve for $\operatorname{det}(Z)=0$, being $Z$ a matrix operator, since $\operatorname{det}(Z)=\prod_{n} \nu_{n}$.

$$
Z \mathbf{I}_{n}(\mathbf{r}, \omega)=\nu_{n} \mathbf{I}_{n}(\mathbf{r}, \omega)
$$

In (4), $\mathbf{I}_{n}$ is the natural basis or the natural modes. It is important to note that both $\mathbf{I}_{n}$ and $\nu_{n}$ are generally complex, except for the related inner solutions. From this natural base, it is possible to construct the total current distribution $\mathbf{J}$ as

$$
\mathbf{J}(\mathbf{r}, \omega)=\sum_{n} \frac{<\mathbf{I}_{n}(\mathbf{r}, \omega), \mathbf{E}^{i}>}{\nu_{n}<\mathbf{I}_{n}(\mathbf{r}, \omega), \mathbf{I}_{n}(\mathbf{r}, \omega)>} I_{n}(\mathbf{r}, \omega)
$$

This method is known as eigenfunction expansion method (EEM). More details can be found in [7].

Total current $\mathbf{J}(\mathbf{r}, \omega)$ can be expanded in terms of so-called characteristic modes as well. When using CMA, it is not necessary to resort to complex frequencies and complex eigenvalues and eigenvectors to find the same $\mathbf{J}(\mathbf{r}, \omega)$. Unlike the physical solutions provided by the EEM, CMA provides a mathematical basis with some interesting properties facilitating their manipulation and understanding when designing antennas or scatterers. CMA exploits the mathematical properties of the $Z$ operator to obtain real eigenvalues and eigenmodes. Since $Z=R+j X$ is a complex symmetric operator, its real and imaginary parts, $R$ and $X$, are hermitian matrices. The following generalized or weighted eigenvalue problem defines the characteristic eigenvalues, $\lambda_{n}$, and the characteristic eigencurrents, $\mathbf{J}_{n}$.

$$
X \mathbf{J}_{n}(\mathbf{r}, \omega)=\lambda_{n} R \mathbf{J}_{n}(\mathbf{r}, \omega)
$$

By definition, the characteristic eigenvalues, $\lambda_{n}$, range from $-\infty$ to $+\infty$. Furthermore, if $\lambda_{n}>0$, those related modes have predominantly magnetic stored energy (inductive modes), while if $\lambda_{n}<0$ they have electric stored energy (capacitive modes). A mode having $\lambda_{n}= \pm \infty$ is called an internally resonant mode (cavity mode). And a mode having $\lambda_{n}=0$ is called an externally resonant mode. The modes corresponding to external resonances are the ones considered in antenna and scatterer design.

Current $\mathbf{J}$ can be obtained from the expansion of their characteristic eigensolutions as

$$
\mathbf{J}(\mathbf{r}, \omega)=\sum_{n} \frac{<\mathbf{J}_{n}(\mathbf{r}, \omega), \mathbf{E}^{i}>}{\left(1+j \lambda_{n}\right)<\mathbf{J}_{n}(\mathbf{r}, \omega), \mathbf{J}_{n}(\mathbf{r}, \omega)>} \mathbf{J}_{n}(\mathbf{r}, \omega)
$$

\section{INFINITE PEC CIRCULAR CYLINDER}

In this section, natural resonances and characteristic resonances for a PEC infinite circular cylinder are compared when considering the EFIE impedance operator. For this purpose, the well known analytical formulas to express the EFIE operator for $T M^{z}$ and $T E^{z}$ polarizations are used [18], [19].

$$
\begin{aligned}
Z^{E F I E, T M_{n}^{z}} & =\frac{\eta \pi k a}{2} J_{n}(k a) H_{n}^{(2)}(k a) \\
Z^{E F I E, T E_{n}^{z}} & =\frac{\eta \pi k a}{2} J_{n}^{\prime}(k a) H_{n}^{\prime(2)}(k a)
\end{aligned}
$$

$a$ is the cylinder radius, $H_{n}^{(2)}(x)$ the Hankel functions of second kind, $J_{n}(x)$ the Bessel functions of first kind, $n$ the azimuthal mode order, $k$ the wavenumber, and $\eta$ the intrinsic impedance. The primes on the Bessel and Hankel functions denote differentiation with respect to the entire argument.

\section{A. Natural Resonances}

Natural resonances are extracted from the zeros of the determinant of the impedance matrix operator. Thus, concerning the PEC infinite circular cylinder, equations (8) and (9) have to be equal to zero. Some of the first natural resonances are shown in Fig. 1.

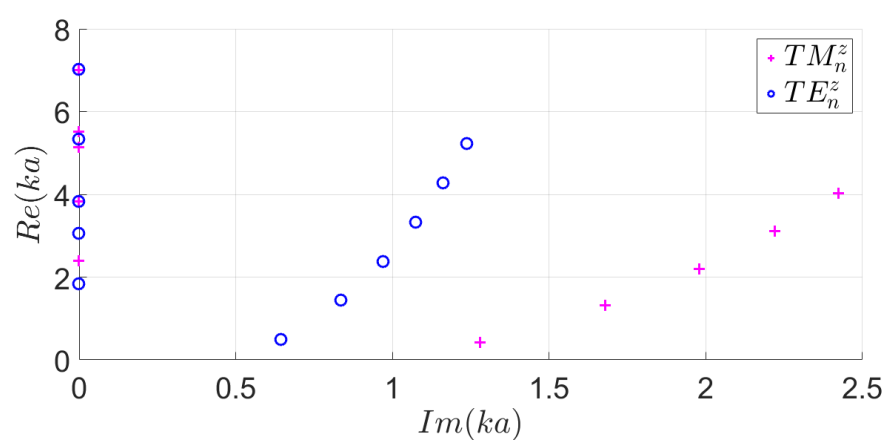

Fig. 1: Natural resonances of the infinite PEC circular cylinder.

$T M_{n}^{z}$ and $T E_{n}^{z}$ resonances are marked with "+" and "o", respectively. Considering the conclusions presented in [20] for the PEC sphere, the same can be concluded here: natural resonances in Fig. 1 can be separated into internal (cavity modes) and external natural resonances (surface modes or creeping waves).

The internal resonances are those located on the real axis and the external ones are complex numbers. The imaginary part of these complex numbers (surface modes) provides surface mode ability to radiate (radiation losses). Unlike the surface modes, cavity modes do not radiate and that is why their resonances are pure real numbers. Just for the sake of completeness, let us add that these cavity resonances are transverse resonances and they are the cut-off frequencies of the corresponding circular waveguide modes, i.e., $J_{n}(k a)=0$ $\left(T M_{n}^{z}\right.$ modes) and $J_{n}^{\prime}(k a)=0$ ( $T E_{n}^{z}$ modes). This means that looking at equations (8) and (9), external resonances can be calculated from $H_{n}^{(2)}(k a)=0$ (T $M_{n}^{z}$ modes $)$ and $H_{n}^{\prime(2)}(k a)=0\left(T E_{n}^{z}\right.$ 
modes). External resonances are due to those surface waves that circumnavigate the cylinder surface matching their phases so as to build up to the resonance by constructive interference.

\section{B. Characteristic Mode Resonances}

CMRs are obtained from the generalized eigenvalue problem (GEP) presented below [9]

$$
X \mathbf{J}_{n}=\lambda_{n} R \mathbf{J}_{n}
$$

where $X$ and $R$ are the imaginary and real part of the EFIE impendance matrix operator $(Z=R+j X)$ respectively, $\mathbf{J}_{n}$ the characteristic currents, and $\lambda_{n}$ the characteristic eigenvalues. By definition, $\lambda_{n}=0$ and $\lambda_{n}= \pm \infty$ (asymptotic behavior) are being interpreted as external and internal characteristic resonances, respectively.

Concerning the infinite PEC cylinder, characteristic eigenvalues can be found substituting equations (8) and (9) into (10). The analytical results for their associated eigenvalues are [8]

$$
\begin{aligned}
& \lambda_{n}^{T M_{n}^{z}}=-\frac{Y_{n}(k a)}{J_{n}(k a)} \\
& \lambda_{n}^{T E_{n}^{z}}=-\frac{Y_{n}^{\prime}(k a)}{J_{n}^{\prime}(k a)}
\end{aligned}
$$

Equations (11) and (12) are graphed in Fig. 2.

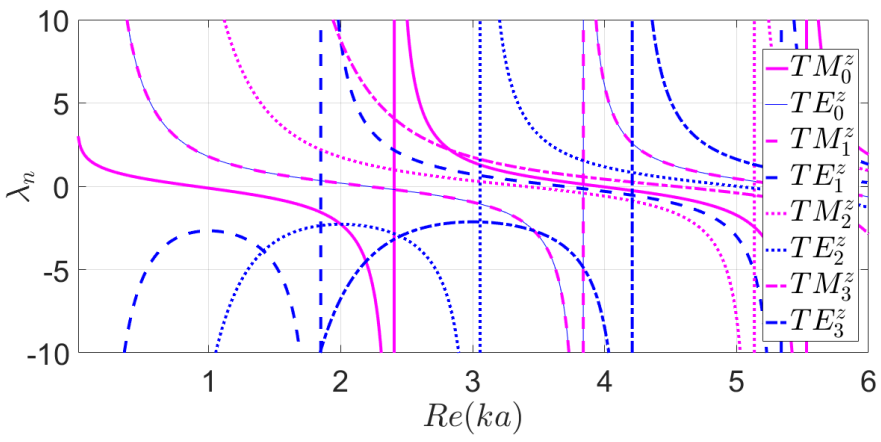

Fig. 2: Characteristic eigenvalues of the infinite PEC circular cylinder.

Let us consider for instance the $T M_{n}^{z}$ characteristic eigenvalues. Observing observing equation (11) in Fig. 2, it can be clearly seen that the zero crossings of $\lambda_{n}$ correspond with the zeros of the Neumann function, $Y_{n}(k a)$. Moreover, when $\lambda_{n}= \pm \infty$ (asymptotic behavior), it corresponds with the zeros of the Bessel function, $J_{n}(k a)$. Therefore, $Y_{n}(k a)=0$ and $J_{n}(k a)=0$ provide the external and internal CMRs, respectively. Furthermore, it is also important to note that in $\lambda_{n}$ curves, each asymptote is located between two consecutive zeros. The consecutive asymptotes provide the order of the radial variation of a given cavity mode, i.e., the corresponding $\lambda_{n}$ for $T M_{0}$ mode has $m=1,2,3 \ldots$ asymptotes that are representing the cavity resonances with radial variations $T M_{0, m}$. They are for instance $T M_{0,1}, T M_{0,2}$, $T M_{0,3}$, and so on. Regarding the zero crossings of $\lambda_{n}$, they are simply solutions related with the zeros of the reactance operator $X$, as a result of using the GEP (2). As mentioned above, since we are dealing with a circular PEC infinite cylinder these zeros are exactly the zeros of the Neumann function. According to CMA, these are called external characteristic resonances. Interestingly, they do not coincide with the external natural resonances, as it will be evidenced next.
TABLE I: Comparison between external natural resonances and external characteristic modes resonances.

\begin{tabular}{|l|ll|ll|}
\hline$R e(k a)$ & Natural Resonances & & Characteristic Mode Res. & $T E_{n, 1}$ \\
\hline$n$ & $T M_{n, 1}$ & $T E_{n, 1}$ & $T M_{n, 1}$ & 2.20 \\
\hline 0 & $\mathrm{X}$ & $\mathrm{X}$ & 0.89 & 3.68 \\
1 & $\mathrm{X}$ & 0.50 & 2.20 & 5.00 \\
2 & 0.43 & 1.44 & 3.38 & $\mathrm{X}$ \\
3 & 1.31 & 2.38 & 4.53 & $\mathrm{X}$ \\
4 & 2.21 & 3.32 & 5.65 & \multicolumn{2}{|c}{} \\
\hline
\end{tabular}

The same can be concluded for the $T E_{n}^{z}$ characteristic modes, but considering equation (12). $Y_{n}^{\prime}(k a)=0$ and $J_{n}^{\prime}(k a)=0$ lead to external and internal CMRs, respectively.

\section{Natural Resonances vs Characteristic Modes Resonances}

With regard to internal resonances, it is obvious that both procedures lead to exactly the same results, since both are calculated from $J_{n}(k a)=0\left(T M_{n}^{z}\right)$ and $J_{n}^{\prime}(k a)=0\left(T E_{n}^{z}\right)$. As for external resonances, Table I shows the corresponding values obtained from Fig. 1 and Fig. 2. Contrary to [15], [16], where it was stated that external CNRs and CMRs were the same, here it is concluded that both methods provide quite different resonances. Notice that letter $\mathrm{X}$ on Table I is for points outside the graph region shown.

Nevertheless, it would be interesting to show whether characteristic eigenvalues $\left(\lambda_{n}\right)$ are somehow capable of providing the external natural resonances. Notice, though, that external CNRs involve complex $k a$ values. However a complex argument should not be used directly on (11) or (12) because these equations were obtained by Garbacz assuming real $k a$ values. That would lead to wrong solutions. Instead, the proper thing to do is introducing complex $k a$ values in (8) and (9) before solving equation (10). It is worth noting that $\lambda_{n}$ remains a real number. The eigenvalues obtained for complex $k a$ are shown in Fig. 3.

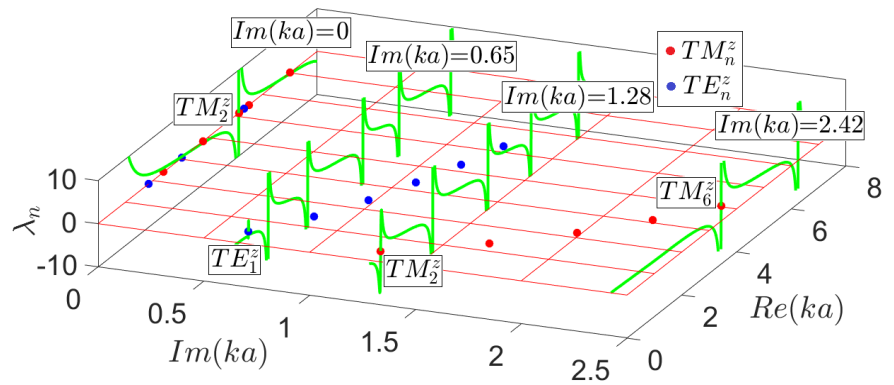

Fig. 3: Characteristic eigenvalues for different imaginary planes and the poles of the natural resonances.

A few resonances have been chosen for the sake of illustration. Fig. 3 shows a schematic correspondence between the characteristic eigenvalues $\left(\lambda_{n}\right)$ obtained for a set of imaginary $k a$ planes and the natural resonance poles for the PEC cylinder. The real part corresponds to the location of the resonant frequencies and the imaginary part to the damping factor. The imaginary $k a$ planes selected contain external natural resonances of modes $T E_{1}^{z}, T M_{2}^{z}$ and $T M_{6}^{z}$. These planes are $\operatorname{Im}(k a)=0.65,1.28,2.42$ (See fig. 1). Notice that each eigenvalue trace crosses zero or have an asymptote right at the natural resonance location. For $\operatorname{Im}(k a)=0$ plane, only the internal natural resonance of $T M_{2}^{z}$ mode matches the eigenvalue trace. Therefore, now characteristic eigenvalues are able to provide the natural resonances. Along with the natural resonances, the $\lambda_{n}$ 
curves plotted, show other zero crossings and asymptotes. These are just due to singularities in $X$ or $R$ operators in equation (10) with no physical meaning, to the authors' knowledge.

\section{GEOMETRICAL RELATION BETWEEN CNR AND CMR}

At this point, it is interesting to explain why external CNRs and external CMRs are different. The electric current $\mathbf{J}$ is related to $\mathbf{E}^{i}$ through the impedance operator $Z$ as

$$
Z(\mathbf{J})=\mathbf{E}^{i}
$$

$Z$ is a symmetric complex matrix operator containing the geometrical and material information about the scatterer under study.

In order to relate natural resonances with the resonances obtained from the characteristic eigenvalues, it is worth having a look to the standard eigenvalues obtained from $Z$. Equation (14) presents the SEP

$$
Z \mathbf{I}_{n}=\nu_{n} \mathbf{I}_{n}
$$

where $\mathbf{I}_{n}$ are the eigencurrents, and $\nu_{n}$ their associated eigenvalues. Since $Z$ is a non-hermitian symmetric matrix, both, eigenvalues and eigenvectors, are generally complex. Since one seeks to solve the nontrivial solution for equation (13), $Z(\mathbf{J})=0$, natural resonances are calculated by forcing the determinant of the impedance matrix operator to vanish, but the same can be achieved from the eigenvalues of the SEP (14) when they are set to zero, i.e., $\nu_{n}=0$. Thus, natural resonances can be found if $\nu_{n}=0$.

Let us consider the $T E_{n}^{z}$ solution of the infinite PEC circular cylinder as an example. Note that since equations (8) and (9) represent a diagonal impedance matrix operator, their associated impedance eigenvalues are exactly the same equations. Then, from the SEP viewpoint we have

$$
\nu_{n}^{T E_{n}^{z}}=\frac{\eta \pi k a}{2} J_{n}^{\prime}(k a) H_{n}^{\prime(2)}(k a)
$$

In order to study the eigenimpedance $\nu_{n}^{T E_{n}^{z}}$, also known as characteristic impedance, it is interesting to separate it into its real and imaginary parts, $\nu_{n}=\alpha_{n}+j \beta_{n}$. Where $\alpha_{n}$ and $\beta_{n}$ represent the resistance and the reactance, respectively. Characteristic impedance is commonly represented in polar coordinates as $\nu_{n}=\left|\nu_{n}\right| e^{j \phi_{n}}$. Where $\left|\nu_{n}\right|=\sqrt{\alpha_{n}^{2}+\beta_{n}^{2}}$, and $\phi_{n}=\arctan \left(\beta_{n} / \alpha_{n}\right)$ is the phase angle. This polar representation is called impedance diagram. Considering the $T E_{1}^{z}$ mode from equation (15), its impedance diagram is presented in Fig. 4.

In Fig. $4, \alpha_{n}$ is plotted on the $\mathrm{X}$-axis and $\beta_{n}$ on the Y-axis. Each point represented by vector $\nu_{n}$ is the characteristic impedance at a given frequency. In Fig. 4, frequency grows in the clockwise direction. As frequency increases, $\nu_{1}$ is generating a circle. When $\nu_{1}=0$ it implies that $\left|\nu_{1}\right|=0$ and $\phi_{1}=\pi / 2$ or $-\pi / 2$. Only in this case (considering real $k a$ values), the EFIE eigenvalue (15) breaks down giving an internal resonance. The internal resonance problem is explained in [21] and [18] from the numerical viewpoint, and it is also explained in [17] for the $T M_{n}^{z}$ polarization considering characteristic modes. On the other hand, $\nu_{1}$ experiences its minimum value $\left(\nu_{1}=\alpha_{1}\right)$ when $\phi_{1}=0$, indicating the cylinder impedance is purely resistive. Moreover, right at this frequency the cylinder is supposed to present a maximum value in its associated eigencurrent under study. Finally, when $\phi_{1}>0$ and $\phi_{1}<0$, the associated eigencurrent is behaving inductively and capacitively, respectively.

The analogy with the generalized equation governing characteristic modes (10) is that since equation (10) can also be expressed as $\alpha_{n} X \mathbf{J}_{n}=\beta_{n} R \mathbf{J}_{n}$, it is straightforward to write $\lambda_{n}=\beta_{n} / \alpha_{n}$.

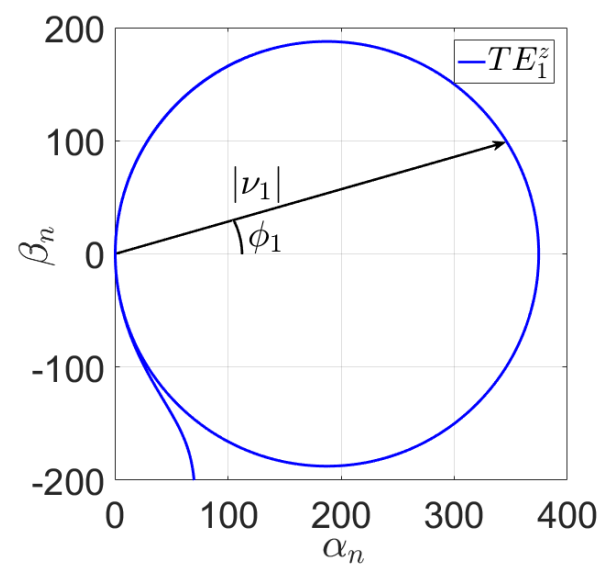

Fig. 4: Impedance diagram for the $T E_{1}^{z}$ eigenimpedance.

According to this, it is concluded that the TCM diagonalizes the phase angle of the characteristic impedance. That is why the information provided by $\lambda_{n}$ is related with the energy storage of the scatterer under study, either inductive or capacitive, and the resonances, as defined in section III-B. This makes clear that the relation between the eigenvalues obtained from the SEP and the GEP is

$$
\nu_{n}=\left|\nu_{n}\right| e^{j \arctan \left(\lambda_{n}\right)}
$$

Somehow Garbacz explained this in his thesis [8], but considering other parameter definitions.

Going forward, the external natural resonances are not presented in Fig. 4 (only the internal ones), unless complex $k a$ values were considered to achieve $\nu_{n}=0$, as demonstrated in Fig.3. Notice that at an external characteristic mode resonance, $\lambda_{n}=0$. Thus, $\phi=0$ and $\nu_{n}=\left|\nu_{n}\right|$ and at its corresponding frequency, the condition $\nu_{n}=0$ is not accomplished and the maximum eigencurrent is not obtained. This simple reasoning contradicts the assumption done by other researchers that characteristic resonances and natural resonances are equal, and that characteristic modes radiate maximum field when they are in resonance.

To finalize this discussion, let us explain what is happening in the total electric current and the scattered electric field at a $k a$ value for which an external characteristic mode resonates. Since external resonances from characteristic eigenvalues do occur at real frequencies for which equations (8) and (9) are not singular, the external natural resonances are not excited, i.e., the singularity condition $\operatorname{det}(Z)=0$ is not met. Thus, the maximum radiated field and maximum current distribution will not be in scattering resonance. To illustrate this point, let us consider the same explanation procedure followed by Sarkar in [17]. As mentioned in the previous section, external characteristic mode resonances for the $T M_{n}^{z}$ polarization occur when $Y n(k a)=0$. Unlike natural resonance which is produced for the complex $k a$ value considering $H_{n}(k a)=0$, if $Y_{n}(k a)=0$ is substituted in equation (17), a maximum electric current does not occur at this $k a$ real value. The same can be said for the scattered field if one looks at equation (18). Only $H_{n}(k a)=0$ breaks down electric current and scattered field.

$$
J_{z}=\frac{-2 E_{0}}{k \eta \pi a} \sum_{n=-\infty}^{\infty} \frac{j^{-n} e^{j n \phi}}{H_{n}^{(2)}(k a)}
$$




$$
E_{z}^{s}=-E_{0} \sum_{n=-\infty}^{\infty} j^{-n} \frac{J_{n}(k a)}{H_{n}^{(2)}(k a)} H_{n}^{(2)}(k \rho) e^{j n \phi}
$$

\section{CONCLUSiON}

In this paper, some aspects in the interpretation of the solutions obtained when analyzing PEC cavities with the TCM have been presented. Firstly, natural resonances and CMRs of the infinite PEC circular cylinder have been presented using the analytical EFIE operators. These operators are those calculated considering both $T M_{n}^{z}$ and $T E_{n}^{z}$ polarizations. Secondly, natural resonances and characteristic mode resonances have been compared. It has been concluded that external natural resonances and external characteristic mode resonances are different. However, internal natural resonances and internal characteristic mode resonances coincide. Moreover, characteristic eigenvalues have been used to find natural resonances in complex $k a$ plane. It has been shown that characteristic eigenvalues remain real while it is possible to locate natural resonances with them. In addition, it has been explained why external natural resonances and external CMRs are different. This has been done using the SEP and the polar representation of the characteristic impedance. It can be concluded that characteristic eigenvalues are related with the phase angle of the characteristic impedance and they do not predict external natural resonances when considering real $k a$ values. Finally, the thesis stating that being at a given external CMR does not imply maximum wave scattering is also demonstrated.

It is worth remarking, however, that the conclusions drawn, apply to a closed conducting body exhibiting internal and external resonances. Open bodies such as flat plates or thin dipoles will not show such important differences between external characteristic resonances and external natural resonances since the imaginary part of the latter are significantly closer to the real axis.

\section{REFERENCES}

[1] C. E. Baum, E. J. Rothwell, K.-M. Chen, and D. P. Nyquist, "The singularity expansion method and its application to target identification," Proceedings of the IEEE, vol. 79, no. 10, pp. 1481-1492, 1991.

[2] D. Moffatt et al., "Natural resonances of radar targets via prony's method and target discrimination," IEEE Transactions on Aerospace and Electronic Systems, no. 5, pp. 583-589, 1976.

[3] T. K. Sarkar and O. Pereira, "Using the matrix pencil method to estimate the parameters of a sum of complex exponentials," IEEE Antennas and Propagation Magazine, vol. 37, no. 1, pp. 48-55, 1995.

[4] W. Lee, T. K. Sarkar, H. Moon, and M. Salazar-Palma, "Computation of the natural poles of an object in the frequency domain using the cauchy method," IEEE Antennas and Wireless Propagation Letters, vol. 11, pp. 1137-1140, 2012.

[5] A. W. Glisson, D. Kajfez, and J. James, "Evaluation of modes in dielectric resonators using a surface integral equation formulation," IEEE transactions on microwave theory and techniques, vol. 31, no. 12, pp. 1023-1029, 1983.

[6] A. A. Kishk, A. Glisson, and D. Kajfez, "Computed resonant frequency and far fields of isolated dielectric discs," in Antennas and Propagation Society International Symposium, 1993. AP-S. Digest. IEEE, 1993, pp. $408-411$.

[7] C. E. Baum, "On the eigenmode expansion method for electromagnetic scattering and antenna problems, part i: some basic relations for eigenmode expansions and their relation to the singularity expansion," Interaction Note, vol. 229, p. 13, 1975.

[8] R. J. Garbacz, "A generalized expansion for radiated and scatered fields," Ph.D. dissertation, The Ohio State University, Columbus, 1968.

[9] R. Harrington and J. Mautz, "Theory of characteristic modes for conducting bodies," Antennas and Propagation, IEEE Transactions on, vol. 19 , no. 5 , pp. $622-628,1971$.
[10] R. Harrington, J. Mautz, and Y. Chang, "Characteristic modes for dielectric and magnetic bodies," Antennas and Propagation, IEEE Transactions on, vol. 20, no. 2, pp. 194-198, 1972.

[11] Y. Chang and R. Harrington, "A surface formulation for characteristic modes of material bodies," Antennas and Propagation, IEEE Transactions on, vol. 25, no. 6, pp. 789-795, 1977.

[12] M. Cabedo-Fabres, E. Antonino-Daviu, A. Valero-Nogueira, and M. F. Bataller, "The theory of characteristic modes revisited: A contribution to the design of antennas for modern applications," Antennas and Propagation Magazine, IEEE, vol. 49, no. 5, pp. 52-68, 2007.

[13] P. D. Larison, "Evaluation of system identification algorithms for aspectindependent radar target classification," DTIC Document, Tech. Rep., 1989.

[14] T. Bernabeu-Jimenez, F. Vico-Bondia, A. Valero-Nogueira, M. CabedoFabres, F. Gallee, and E. Antonino-Daviu, "Understanding the analytical formulation of the characteristic modes," in IEEE International Symposium on Antennas and Propagation and USNC-URSI National Radio Science Meeting, 2013. IEEE, 2013, p. 526.3.

[15] H. Alroughani, "An appraisal of the characteristic modes of composite objects," Master thesis, University of Ottawa, 2013.

[16] R. Rezaiesarlak and M. Manteghi, "Design of chipless rfid tags based on characteristic mode theory (cmt)," IEEE Transactions on Antennas and Propagation, vol. 63, no. 2, pp. 711-718, 2015.

[17] T. K. Sarkar, E. L. Mokole, and M. Salazar-Palma, "An expose on internal resonance, external resonance, and characteristic modes," IEEE Transactions on Antennas and Propagation, vol. 64, no. 11, pp. 46954702, 2016.

[18] K. F. Warnick, Numerical analysis for electromagnetic integral equations. Artech House, 2008.

[19] A. F. Peterson, S. L. Ray, R. Mittra, I. of Electrical, and E. Engineers, Computational methods for electromagnetics. IEEE press New York, 1998, vol. 2.

[20] C.-C. Chen, "Electromagnetic resonances of immersed dielectric spheres," Antennas and Propagation, IEEE Transactions on, vol. 46, no. 7, pp. 1074-1083, 1998

[21] A. J. Poggio and E. K. Miller, Integral equation Solution of Threedimensional Scattering Problems, Computer techniques in electromagnetics, R. Mittra. Pergamon Press, Oxford, pp. 159-261, 1973. 